\title{
Annealing Effect on the Formation of In(Ga)As Quantum Rings From InAs Quantum Dots
}

\author{
Jong-Horng Dai, Jheng-Han Lee, and Si-Chen Lee, Fellow, IEEE
}

\begin{abstract}
The correlation between the In(Ga)As quantum rings (QRs) and their precursors InAs quantum dots (QDs) are studied by changing the annealing time after the deposition of a thin InAs layer on GaAs. The atomic force microscopy and photoluminescence (PL) are used to characterize the morphologies and electronic states of QDs and QRs. The longer annealing time tends to enlarge the QD size to high aspect ratio, group InAs islands into several different sizes, and red-shift the PL peak. The resultant QRs show the ridge-confined rings and the PL peaks of QRs blue-shift with increasing QD aspect ratio. It demonstrates an important way to tailor the electronics states of QRs.
\end{abstract}

Index Terms-Adatom migration, annealing time, elastic relaxation, quantum dots (QDs), quantum rings (QRs).

\section{INTRODUCTION}

$\mathbf{T}$ HE self-assembled InAs quantum dots (QDs) grown on GaAs substrate by molecular beam epitaxy (MBE) have been widely studied in recent years [1]-[3]. The zero-dimensional InAs QDs grown by MBE give a period of annealing time after the deposition of thin InAs layer in the conventional method [4]. Many physical and electronic properties of QDs, i.e., dot size, density, distribution, and bandgap are varied during the annealing [5]. These QDs are typically not uniform in size, the quantum ring $(\mathrm{QR})$ formed by capped $\mathrm{QD}$ with a thin GaAs layer and subsequent annealing can solve the problem [6]. The formation of QR is one method of size-limited InAs island growth which is a nonequilibrium process resulting from diffusion of In atoms from the uncovered InAs QDs and wetting of the surrounding solid-phase GaAs [7]. Therefore, the optical and electrical properties of the $\mathrm{QR}$ are similar to those of the small QDs and have strong correlation with the precursors QD characteristics. However, the systematic studies of the processing parameters which illustrate the formation of QR from QD are still lacking. In this letter, the effect of annealing on the height, diameter, density, and optical properties of the QDs and QRs were investigated utilizing atomic force microscopy (AFM), cross-sectional transmission electron microscopy (TEM), and photoluminescence (PL) measurements. The growth mechanisms that correlate the annealing condition

Manuscript received July 2, 2007; revised October 22, 2007. This work was supported by the National Science Council of the Republic of China, Taiwan, under Contract NSC-95-2120-M-002-007.

The authors are with the Department of Electrical Engineering and Graduate Institute of Electronics Engineering, National Taiwan University, Taipei 10617 Taiwan, R.O.C.

Color versions of one or more of the figures in this letter are available online at http://ieeexplore.ieee.org.

Digital Object Identifier 10.1109/LPT.2007.912481

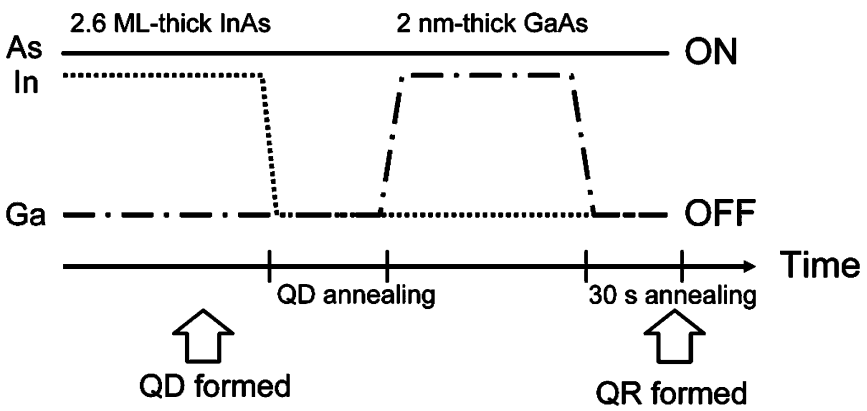

Fig. 1. Shutter opening sequence for MBE growth of QDs and QRs.

with the formation of QRs from QDs were demonstrated and discussed.

\section{EXPERIMENTS AND RESULTS}

The QD and QR were grown on semi-insulating (100) GaAs substrates using VG V80H MKII solid source MBE equipped with valved cracker sources under an $\mathrm{As}_{2}$ beam equivalent pressure of $\sim 3 \times 10^{-6} \mathrm{mbar}$ and V/III ratio of $\sim 1.5$. The structures consisted of a 200-nm GaAs buffer layer, a QD/QR layer, a 60-nm-thick GaAs barrier layer, and a surface $\mathrm{QD} / \mathrm{QR}$ layer. The surface QD/QR layer was grown under the same condition as the buried QD/QR layer for AFM measurement. The buried QD/QR layer was for PL measurement. The first GaAs buffer layer was grown at $600{ }^{\circ} \mathrm{C}$ at a growth rate of $1 \mu \mathrm{m} /$ hour. The temperature was then lowered to $500^{\circ} \mathrm{C}$ to grow 2.2 monolayers (MLs) InAs QD at a growth rate of $0.2 \mathrm{ML} / \mathrm{s}$. After growth of QDs, the In shutter was closed and the samples were annealed at the same temperature for a period of time defined to be the QD annealing time, i.e., 10, 20, 30, and $50 \mathrm{~s}$ and the samples are called D1, D2, D3, and D4, respectively. The shutter opening sequence is shown in Fig. 1. The QR samples were grown by depositing 2-nm GaAs capped layer at $500{ }^{\circ} \mathrm{C}$ on InAs QDs. Then the Ga shutter was closed and the sample was annealed at the same temperature for $30 \mathrm{~s}$ defined to be the QR annealing time. During this time, the center of the most QDs which uncovered by the 2-nm GaAs capped layer diffuse out and mix up with the surrounding GaAs capped layer to form the $\operatorname{In}(\mathrm{Ga}) \mathrm{As}$ ring structure. There is no significant interdiffusion effect in this temperature. The QD annealing time after the growth of QDs for these QR samples are 20,30,40, 50, and $60 \mathrm{~s}$ and the samples are called R1, R2, R3, R4, and R5, respectively. All the sample heaters were turned off the power immediately after the annealing time for reducing the cooling effect resulting in different surface morphologies between surface and buried QDs [2]. The systematic AFM studies on surface and buried InAs QDs by selective removal of the GaAs barrier layer indicated 


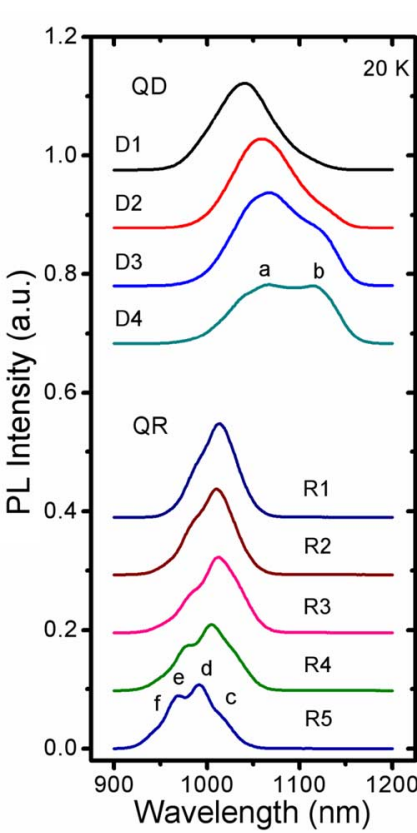

(a)

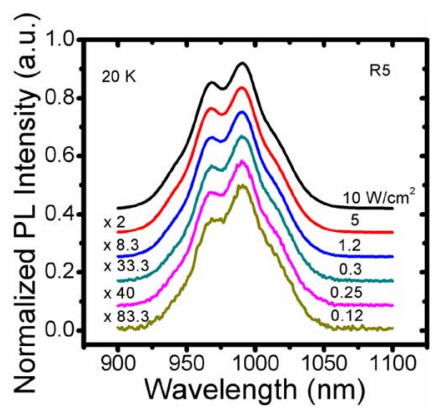

(b)

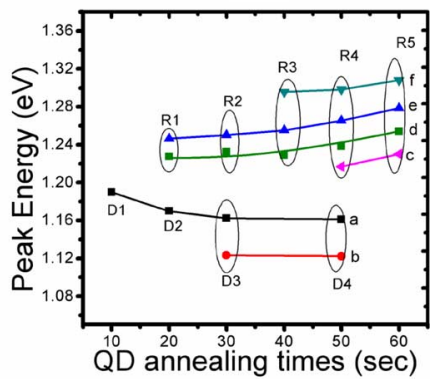

(c)
Fig. 2. (a) PL spectra of two series of QD and QR samples grown at different QD annealing time. (b) Excitation intensity dependence of normalized PL spectra of In(Ga)As QR (sample R5). (c) The dependence of the PL peak energies of two series QR and QD samples on the QD annealing time.

that both buried and surface QDs have similar shape and size distribution. Only the density of the surface QDs is 20\% lower than that of the buried QDs. This could be attributed to a little more prolonged equivalent QD annealing time for surface QDs, i.e., $\sim 5 \mathrm{~s}$.

The PL was excited using an $\mathrm{Ar}^{+}$laser at a 514.5-nm wavelength. The luminescence is dispersed by a $0.5-\mathrm{m}$ triple grating monochromator. The $20 \mathrm{~K} \mathrm{PL}$ spectra of two series of QD and QR samples are shown in Fig. 2(a). It is obvious from the figure that the PL peak wavelength of QDs red-shifts as the QD annealing time increases which leads to a higher QD and lower confined electronic state energy. However, the PL peak wavelength of QRs keeps the same at a shorter wavelength of $1014 \mathrm{~nm}$ for samples R1 to R3 and slightly blue-shifts at a longer annealing time for R4 and R5. Comparing the spectra of samples D2 and R1 with the same 20-s QD annealing time, the full-width at half-maximum (FWHM) of the PL spectrum (R1) of QR is only $49 \mathrm{meV}$, much narrower than that of the QD sample D2, i.e., $\sim 88 \mathrm{meV}$. It is obvious that the numbers of PL peaks depend on the QD annealing time in both series. The multipeak samples as D3, D4, R1 to R5 have been analyzed by measuring PL spectra under different excitation intensity from 0.12 to $10 \mathrm{~W} / \mathrm{cm}^{2}$ at $20 \mathrm{~K}$. One example of sample R5 is shown in Fig. 2(b). The intensity ratio between different peaks is independent on the laser intensity indicating they belong to different size groups. During long QD annealing time, the size distribution of QD is broad. In Fig. 2(a), the two peaks in QD series are labeled as $a$ and $b$, and the four peaks in QR series are labeled as $c, d, e$, and $f$. The dependence of peak energies of two series of samples as a function of QD annealing time is shown in Fig. 2(c). The PL peak energies in the same series show the same tendency of variation when QD annealing time

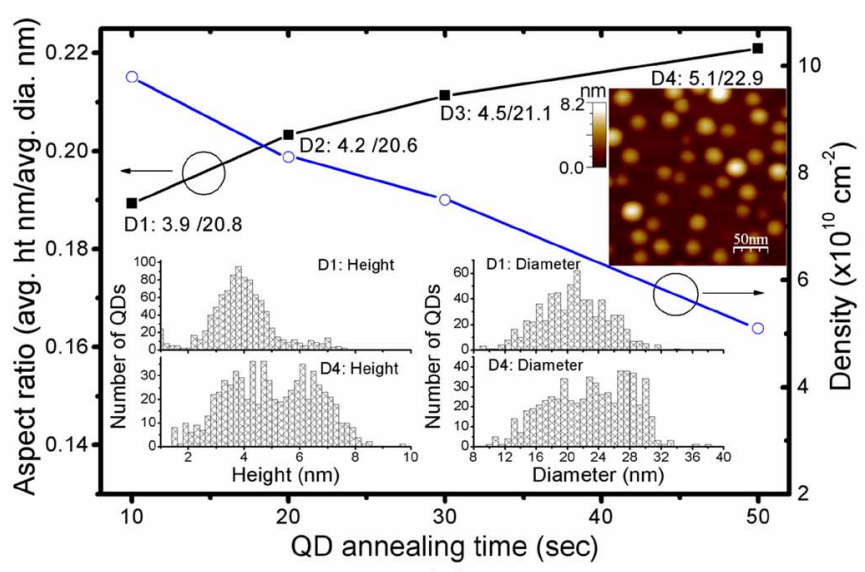

(a)

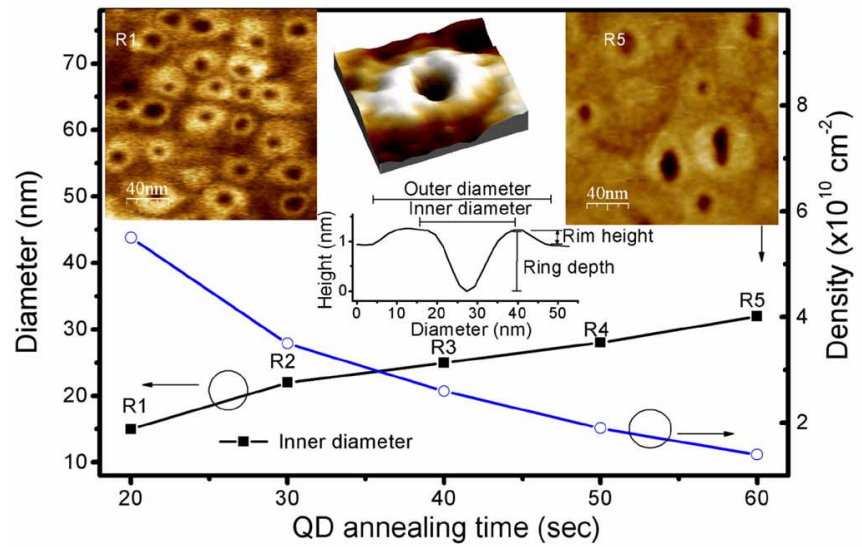

(b)

Fig. 3. (a) Aspect ratios and densities of QDs as a function of annealing time measured by AFM. Insets are the sample D4 image $(250 \mathrm{~nm} \times 200 \mathrm{~nm})$ and the height and diameter histograms of the InAs QDs annealed for $10 \mathrm{~s}$ (sample D1) and $50 \mathrm{~s}$ (sample D4). (b) Inner diameters and densities of QRs as a function of QD annealing times measured by AFM. In the insets, the $200 \mathrm{~nm} \times 200 \mathrm{~nm}$ AFM images of $\operatorname{In}(\mathrm{Ga}) \mathrm{As}$ QRs annealed at $20 \mathrm{~s}$ (sample R1) and $60 \mathrm{~s}$ (sample $\mathrm{R} 5)$, the single $\mathrm{QR}$ image and the contour line are from sample R1.

increases. It is worth mentioning here that QR PL peak energies shift in opposite ways to those of the QD samples when the QD annealing time increases. The reason will be discussed later.

\section{ANALYSIS AND DISCUSSION}

Fig. 3(a) shows the aspect ratio and density of QDs in samples series $D$ measured by AFM. The analyses were performed by a digital instrument multimode AFM using the tapping mode. The histograms showing the diameter and height of QDs in samples D1 and D4 are displayed in the inset. The aspect ratios of the QDs are defined to be the average dot height divided by average dot diameter which is statistically enumerated over several hundred QDs which calculate coherent islands only [4]. The average diameters of samples from D1 to D4 are 20.8-22.9 nm. The diameter distribution which is defined to be the standard deviation divided by mean of the QD diameter (= sd of dia./mean) is 0.21 for sample D1 which is not far from 0.23 for sample D4. Contrary to the diameter, the average height of QDs increases with increasing annealing time, i.e., from $3.9 \mathrm{~nm}$ for D1 to $5.1 \mathrm{~nm}$ for D4. The height of sample D1 has a standard deviation of $1.17 \mathrm{~nm}$ which is smaller than that of sample D4, $1.86 \mathrm{~nm}$. Hence, the 


\section{InAs QD with $2 \mathrm{~nm}$ GaAs capped layer}

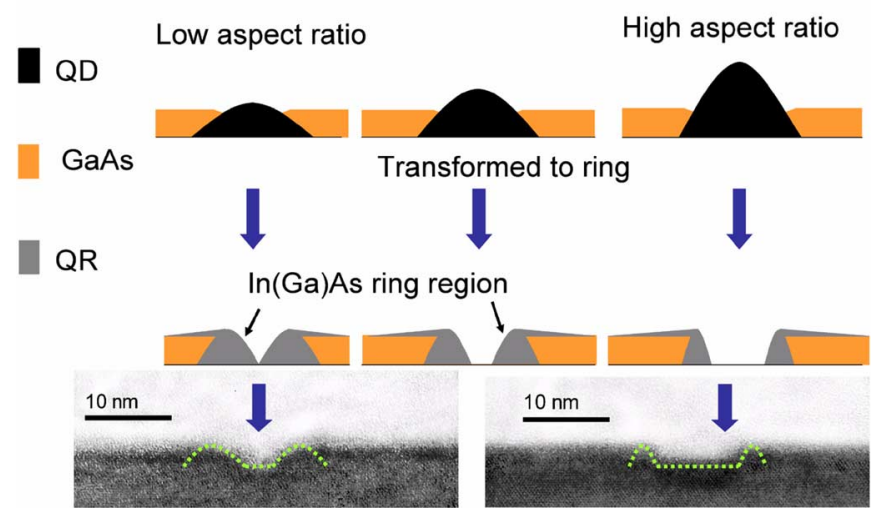

Fig. 4. Model to explain the formation of QRs from QDs with three different sizes and the cross-sectional TEM image of large and small $\mathrm{In}(\mathrm{Ga})$ As ring size from sample R5.

height distribution (= sd of ht/mean) is 0.28 for sample D1 and 0.36 for sample D4 which are larger than diameter distribution. It shows that the size of QDs is very uniform, dense, and coherent for shorter annealing time. The lower density and large variation of QD size in sample D4 is attributed to the fact that some of In adatoms are migrated, desorbed from one dot and incorporated into other dots and grouped into multisized distribution during the QD annealing time [8]. Finally, the average aspect ratio (= avg. ht/avg. dia.) increases from 0.19 to 0.22 with the increasing annealing time from 10 to $50 \mathrm{~s}$. Due to the fact that the strain is largely relaxed on the top of the large QDs during the QD annealing time [3], the growth rate $(0.2 \mathrm{ML} / \mathrm{s})$ is quick, the In adatoms tend to move to the relaxed region which causes the increase of the aspect ratio. The AFM analysis of the inner ring diameter and density of QR samples R1 to R5 are shown in Fig. 3(b). It is observed that the ring depth is limited by 2-nm GaAs capped layer and the rim height which appears on the AFM image as ring shape is only two angstroms [9]. The main $\operatorname{In}(\mathrm{Ga}) \mathrm{As}$ area is buried under the GaAs capped layer for at least $2 \mathrm{~nm}$ deep that provides a confining potential which is better than the surface rim height only confined in a few angstroms [9]. Therefore, the inner diameter is close to the real QR size.

According to the AFM measurement of QDs, it is consistent with PL measurement showing broadened and red-shifted peaks. It is reasonable that the multisized QD group could be transformed to the multisize QR group. The longer annealing time leading to more blue-shifted PL peaks of QR is related to the increasing of the QD aspect ratio. The 2-nm-thick GaAs capped layer covers around the QD hillside; hence, the shape of the QD determines the final morphology of the QR as shown in Fig. 4. The top row of the schematic drawings illustrates the QDs with different aspect ratio. The same 2-nm-thick GaAs capped layer on QDs results in different InAs buried regions. The small QD has the larger buried region and the high aspect ratio QD has less. After out diffusion of InAs from central part of QD, the QR is formed. The $\operatorname{In}(\mathrm{Ga})$ As ring is smaller in size when QD has high aspect ratio. Finally, we examined the sample R5 structure in TEM images. The cross-sectional TEM measurements were performed using a Philips Tecnai F30 microscope with acceleration voltage $300 \mathrm{kV}$. The images show the ring structure above the wetting layer; it is clear that the confining region of rings depends on the aspect ratio of precursor dots. The higher aspect ratio dot results in a smaller confined ring.

\section{CONCLUSION}

The effect of QD annealing time on the morphology and PL spectra of QDs and QRs are investigated. The longer QD annealing time leads to relieve strain elastically in the higher aspect ratio of entire QDs and to enlarge the size distribution. That results in the related PL spectra are broaden and a red-shift. The annealing of QD capped by 2-nm GaAs layer leads to QR segregating into a different size group which is determined by the QD aspect ratio. The PL spectra exhibit multipeaks each with narrower FWHM, and the peak energies blue-shift. These multisize properties of QRs could be employed in the optoelectronic device for multicolor sensor [10].

\section{ACKNOWLEDGMENT}

The authors thank H. R. Chen and Dr. T. Y. Wang from the Department of Materials Science and Engineering for their helpful discussion on TEM analysis.

\section{REFERENCES}

[1] Y. T. Dai, J. C. Fan, Y. F. Chen, R. M. Lin, S. C. Lee, and H. H. Lin, "Temperature dependence of photoluminescence spectra in InAs/GaAs quantum dot superlattices with large thicknesses," J. Appl. Phys., vol. 82, no. 9, pp. 4489-4492, Nov. 1, 1997.

[2] N. N. Ledentsov, V. A. Shchukin, D. Bimberg, V. M. Ustinov, N. A. Cherkashin, Y. G. musikhin, B. V. Volovik, G. E. Cirlin, and Z. I. Alferov, "Reversibility of the island shape, volume, and density in Stranski-Krastanow growth," Semicond. Sci. Technol., vol. 16, pp. 502-506, 2001.

[3] J. Tersoff and F. K. LeGoues, "Competing relaxation mechanisms in strained layers,” Phys. Rev. Lett., vol. 72, no. 22, pp. 3570-3573, May. 1994.

[4] N. K. Cho, S. P. Ryu, J. D. Song, W. J. Choi, J. I. Lee, and H. Jeon, "Comparison of structural and optical properties of InAs quantum dots grown by migration-enhanced molecular-beam epitaxy and conventional molecular-beam epitaxy," Appl. Phys. Lett., vol. 88, pp. 133104 1-133104 3, 2006.

[5] K. Takehana, F. Pulizzi, A. Patane, M. Henini, P. C. Main, L. Eaves, D. Granados, and J. M. Garcia, "Controlling the shape of InAs self-assembled quantum dots by thin GaAs capping layers," J. Cryst. Growth, vol. 251, pp. 155-160, 2003.

[6] K. Park, P. Moon, E. Ahn, S. Hong, E. Yoon, J. W. Yoon, H. Cheong, and J. P. Leburton, "Effects of thin GaAs insertion layer on InAs/(In$\mathrm{GaAs}) / \mathrm{InP}(001)$ quantum dots grown by metalorganic chemical vapor deposition," Appl. Phys. Lett., vol. 86, pp. 223110 1-223110 3, 2005.

[7] A. Lorke, R. Blossey, J. M. Garcia, M. Bichler, and G. Abstreiter, "Morphological transformation of $\operatorname{In}_{y} \mathrm{Ga}_{1-y}$ As islands, fabricated by Stranski-Krastanov growth," Mater. Sci. Eng., vol. B88, pp. 225-229, 2002.

[8] N. P. Kobayashi, T. R. Ramachandran, P. Chen, and A. Madhukar, "In situ, atomic force microscope studies of the evolution of InAs three-dimensional islands on GaAs(001)," Appl. Plys. Lett., vol. 68, pp. 3299-3301, 1996.

[9] P. Offermans, P. M. Koenraad, J. H. Wolter, D. Granados, J. M. Garcia, V. M. Fomin, V. N. Gladilin, and J. T. Devreese, "Atomic-scale structure of self-assembled In(Ga)As quantum rings in GaAs," Appl. Phys. Lett., vol. 87, no. 13, pp. 131902 1-131902 3, 2005.

[10] Z. Chen, E. T. Kim, and A. Madhukar, "Normal-incidence voltage-tunable middle- and long-wavelength infrared photoresponse in self-assembled InAs quantum dots," Appl. Phys. Lett., vol. 80, pp. 2490-2492, 2002. 